\section{$\underset{\substack{\text { hommes } \\ \text { \& migrations }}}{ }$}

Hommes \& migrations

Revue française de référence sur les dynamiques

migratoires

$1301 \mid 2013$

Migrations et mondes ruraux

\title{
Trajectoires migratoires dans l'économie forestière de montagne
}

Du XIX ${ }^{\mathrm{e}}$ siècle à nos jours

\section{Philippe Hanus}

\section{(2) OpenEdition \\ 1 Journals}

\section{Édition électronique}

URL : http://journals.openedition.org/hommesmigrations/1930

DOI : 10.4000/hommesmigrations.1930

ISSN : 2262-3353

\section{Éditeur}

Musée national de l'histoire de l'immigration

\section{Édition imprimée}

Date de publication : 1 janvier 2013

Pagination : 149-156

ISBN : 978-2-919040-21-6

ISSN : 1142-852X

\section{Référence électronique}

Philippe Hanus, «Trajectoires migratoires dans l'économie forestière de montagne », Hommes \& migrations [En ligne], 1301 | 2013, mis en ligne le 31 décembre 2015, consulté le 03 mai 2019. URL : http://journals.openedition.org/hommesmigrations/1930; DOI : 10.4000/hommesmigrations. 1930 


\title{
TRAJECTOIRES MIGRATOIRES DANS L'ÉCONOMIE FORESTIĖRE DE MONTAGNE DU XIXE SIĖCLE À NOS JOURS
}

par PHILIPPE HANUS, fchercheur associé au Laboratoire de recherche historique Rhône-Alpes (LARHRA), UPMF Grenoble

\author{
Dans le domaine de l'immigration rurale, les bûcherons restent \\ quelque peu méconnus. Pourtant, depuis la fin du XIXe siècle, \\ les entreprises forestières des Alpes font appel à d'importants \\ contingents de migrants pour cela. Autrefois dominé \\ par les Italiens, ce marché s'est ouvert à de nouveaux migrants \\ avec l'élargissement de l'Union européenne et la pression \\ de la concurrence internationale. Face à un travail harassant \\ et dangereux, la main-d'œuvre étrangère est soumise \\ à de nombreuses irrégularités.
}

\section{En dépit de l'ancienneté de la présence d'une main-d'œuvre étrangère occupée aux travaux des bois et des champs, l'attention a été peu portée sur cet aspect de l'immigration dans le monde rural.}

Décrivant en 1949 l'activité forestière dans le massif jurassien, le géographe ruraliste René Lebeau fait sienne la traditionnelle opposition entre le paysan sédentaire et le migrant forestier : "Métier de semi-nomade, qui exerce une curieuse attirance, développe un état d'esprit un peu spécial, et relâche parfois les liens familiaux. Il est donc indéniable que l'exploitation des sapins est à l'origine d'un genre de vie très particulier ${ }^{1}$."

Cet "homme des bois" est étranger au pays, édifie sa baraque sur le site d'abattage à l'écart du village et travaille comme un forcené du matin au soir. Il repart avec son modeste pécule une fois la saison terminée et on l'oublie aussitôt. Il ne laisse guère plus de traces dans la documentation des archives et chez les historiens davantage préoccupés par l'installation des immigrés dans les villes.

En dépit de l'ancienneté de la présence d'une maind'œuvre étrangère occupée aux travaux des bois et des champs, l'attention a été peu portée sur cet aspect de l'immigration dans le monde rural ${ }^{2}$. Une des raisons majeures du peu d'intérêt qui lui est manifesté réside probablement dans son invisibilité : de maigres effectifs disséminés dans les fermes, les cabanes d'alpage (bergers transhumants) ou dans la forêt, à l'inverse des cohortes d'ouvriers concentrés en des lieux aisément repérables : usines et faubourgs urbains. En outre, le personnel 
extrêmement mobile de ces chantiers forestiers est difficile à évaluer par les agents recenseurs et échappe donc fréquemment à la raison statistique - a fortiori lorsque les migrants ne sont pas en règle vis-à-vis de la préfecture. Comme le souligne AnneMarie Faidutti-Rudolph, l'activité forestière est un monde particulièrement opaque: "Travaillant en équipe, dans des coupes iso-

À l'aube du XXe siècle, les marchands de bois sollicitent

à l'étranger (Lombardie et Tessin) des équipes de travailleurs spécialisés dans l'activité forestière, accompagnés d'un jeune apprenti (le bocia en dialecte bergamasque) qui s'occupe des tâches liées à la vie domestique. ceux qui ont vécu ou observé cette expérience du chantier en forêt. Une attention particulière a été accordée aux filières de recrutement professionnel, formelles ou informelles, à l'expérience migratoire et aux conditions de travail. En conclusion, nous interrogeons certaines représentations du migrant forestier dans des œuvres artistiques particulièrement édifiantes.

\section{Anciennes migrations saisonnières transalpines}

Dans la France rurale de l'Ancien Régime, les migrations de travail sont importantes ${ }^{4}$. De nombreuses industries (forges, verreries) exigent une main-d'œuvre de spécialistes venus parfois de loin. Ces entreprises ne peuvent toutefois se passer de l'aide des migrants temporaires recrutés pour le ravitaillement en combustibles forestiers. Si l'on s'intéresse de près au parcours des métallurgistes, on laisse souvent de côté les "auxiliaires obscurs" que sont les bûcherons, les charbonniers et les voituriers auxquels les textes ne font allusion que de manière accidentelle. Jusqu'à la seconde moitié du XIXe siècle, la séparation entre migrations intérieures et migrations internationales n'étant pas encore bien établie ${ }^{6}$, des groupes professionnels - aux origines géographiques bien déterminées comme les charbonniers de Novare ou de Bergame - traversent la "frontière alpine" pour faire la saison en France. Ce phénomène migratoire ancien prend, à partir des années 1875 , sa physionomie moderne de mouvement de masse.

La grande crise européenne de la fin du XIX ${ }^{e}$ siècle met fin au système de la pluri-activité rurale et accentue un mouvement de dépopulation des campagnes amplifié par la deuxième révolution industrielle? $e^{7}$ En corollaire, la chute des prix du bois provoque une crise de vocation chez les ouvriers forestiers. Dans ce contexte, les journaliers bûcherons et débardeurs cèdent progressivement la place, pour les travaux forestiers de grande envergure, à des salariés payés à la tâche (tâcherons).

À l'aube du XXe siècle, les marchands de bois sollicitent à l'étranger (Lombardie et Tessin) des équipes - essentiellement masculines - de travailleurs spécialisés dans l'activité forestière, accompagnés d'un jeune apprenti (le bocia en dialecte bergamasque) qui s'occupe des tâches liées à la vie domestique. Ces ouvriers prennent l'exploitation des coupes en marché et s'engagent à livrer au marchand de bois, à une date déterminée, un certain nombre de mètres cubes de grumes, contre le règlement du prix des services en fin de chantier. Ceux-ci savent abattre et façonner les grands résineux à la hache et à la scie, lancer et rassembler ces arbres dans des parcelles incommodes. 
Chantier de bûcherons italiens des Papeteries de France à Saint-Agnan-en-Vercors, vers 1920 (c) D.R.

L'émigration des Transalpins est d'abord saisonnière, de Pâques à la Toussaint, assortie d'un retour au pays natal en hiver. Elle se transforme parfois en un projet d'installation définitive en France au bout de quelques années de migration transfrontalière. Ces déplacements professionnels sont orchestrés depuis la communauté villageoise : les plus expérimentés, au terme de plusieurs saisons en France, encadrent le déplacement des plus jeunes, permettant leur insertion dans les réseaux de travail.

\section{Encadrement institutionnel et réseaux professionnels}

Dans l'Hexagone, les bûcherons se déplacent d'un massif forestier à l'autre, au terme de la campagne. Ces mouvements s'expliquent par le fait que les entreprises achètent des coupes dans un périmètre s'étendant jusqu'à $150 \mathrm{~km}$ de leur lieu d'implantation, comme la scierie Guy à Montréal-La Cluse (Ain) qui exploite des forêts en Haute-Savoie. Cet élargissement de la mise en marché des parcelles forestières donne du fil à retordre aux pouvoirs publics obsédés par le "paradigme de la trace" mais incapables de localiser précisément les migrants. Un ouvrier peut effectivement se trouver sur un chantier éloigné de la commune de domiciliation de l'entreprise qui l'emploie, puis migrer vers une autre concession distante de plusieurs dizaines de kilomètres, à l'instar de G. C. et P. P., deux Bergamasques ayant rempli, au printemps 1917, une déclaration de résidence à Jougne (Doubs), "signalés le 12 octobre dernier par Monsieur le préfet du Doubs comme sétant rendus à Sapois (Jura) et qui n'ont pas paru dans cette commune. Toutes les recherches faites n'ont pu faire découvrir la résidence actuelle de ces deux étrangers". Durant l'entre-deux-guerres, l'industrie du sciage et celle du papier se livrent une 
concurrence impitoyable pour l'approvisionnement en denrées ligneuses et pour le recrutement des travailleurs étrangers. Les chantiers d'approvisionnement des papeteries en plein essor nécessitent l'emploi de forts contingents de bûcherons - de 600 à 700 hommes pour les seules Papeteries de France de Domène (Isère) ${ }^{8}$. Le recrutement s'effectue sous la houlette du patronat, en lien avec les acteurs institutionnels comme le Service de la main-d'œuvre agricole $(\mathrm{SMOA})^{9}$. L'encadrement des équipes est confié à un capo maestro d'origine italienne, attaché depuis longtemps à la même entreprise. Généralement, celui-ci engage les bûcherons dans sa région natale par interconnaissance ${ }^{10}$. Ce contremaitre joue un rôle essentiel dans l'organisation de l'émigration : il est bilingue, fait l'intermédiaire entre pays de départ et pays d'accueil et connaît les démarches administratives.

En 1927, dans un contexte de tensions diplomatiques entre puissances européennes, Mussolini ferme officiellement les frontières, au grand désarroi des fonctionnaires français, comme le directeur de

L'encadrement des équipes est confié à un capo maestro d’origine italienne, attaché depuis longtemps à la même entreprise. Généralement, celui-ci engage les bûcherons

dans sa région natale par interconnaissance. rons en Yougoslavie, en Estonie et en Bulgarie." Malgré ces solutions de remplacement, dans les zones de montagne, les exploitants forestiers vont favoriser l'émigration clandestine des équipes de bûcherons transalpins. Les agents de la préfecture régularisent ensuite, non sans une certaine bienveillance, Bûcherons basques aux Écouges (Vercors), vers 1960. (C) Fonds AndRE VALERY

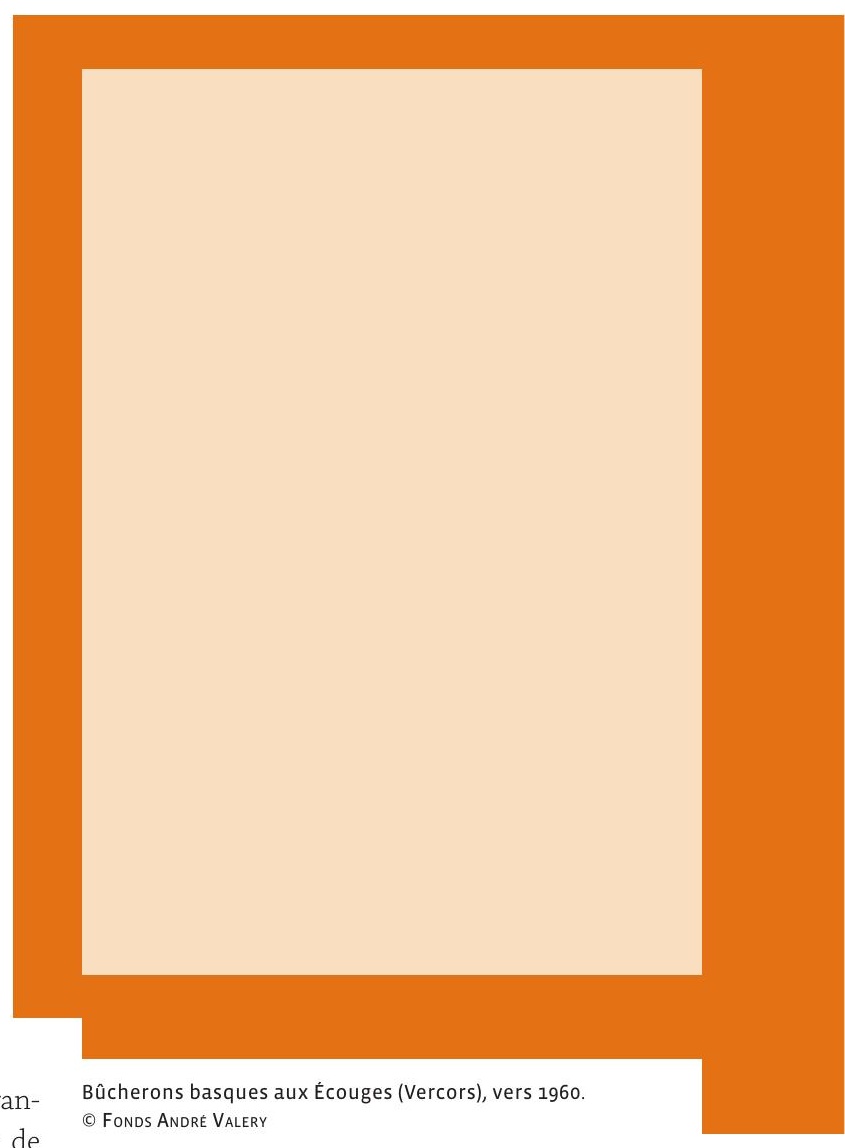

à la bonne marche de l'économie alpine. Un observateur constate en 1933 que, dans toutes les régions montagneuses de l'Isère, "les coupes ne pourraient pas être exploitées si l'emploi des ouvriers étrangers était interdit. En Chartreuse, tous les bûcherons sont italiens. En Oisans, il en est presque de même [...]. Dans les forêts de Belledonne, on compte trois quarts d'Italiens et un quart de gens du pays ${ }^{11 "}$. Il arrive néanmoins que, dans un contexte de chômage élevé et de montée de la xénophobie, des incidents se produisent comme en 1938 dans le massif du Bugey (Ain), lorsque des ouvriers français, délégués par les syndicats, menacent des bûcherons pour qu'ils respectent la loi des 40 heures 
hebdomadaires. Malgré la fermeture des frontières par le régime fasciste, la main-d'œuvre italienne constitue encore, quand la guerre éclate, près de $50 \%$ des effectifs de travailleurs forestiers des Alpes françaises.

\section{De nouveaux travailleurs étrangers en forêt}

Au cours de la Seconde Guerre mondiale, aux côtés des Italiens, des ouvriers requis au titre de la main-d'œuvre indigène dans les colonies, ainsi que des Allemands, Espagnols, Tchèques et Juifs de diverses nationalités opposés à la montée des États fascistes et contraints à l'exil, vont également travailler en forêt $\mathrm{t}^{12}$. Certains d'entre eux, surveillés par les gendarmes, sont occupés au forestage et à la cuisson du charbon de bois dans les groupements de travailleurs étrangers (GTE).

$\mathrm{Au}$ lendemain de la guerre, dans une France exsangue, les vastes chantiers de la reconstruction nationale nécessitent le recours à la main-d'œuvre étrangère, en particulier italienne, dans les mines, le bâtiment et l'exploitation forestière. En ce qui concerne la voie légale, l'introduction en France s'effectue désormais par contrats conclus sous l'égide de l'Office national d'immigration (ONI) qui procède à la sélection des "bons candidats" en Italie ${ }^{13}$. II existe, parallèlement au système de sélection étatique critiqué pour sa lourdeur bureaucratique, une voie irrégulière que vont emprunter environ $40 \%$ des candidats au passage ${ }^{14}$. Entre 1945 et 1950, ce sont des milliers de Transalpins qui franchissent illégalement la frontière alpine, avec ou sans le concours de passeurs ${ }^{15}$, pour rejoindre l'Hexagone, munis parfois d'une simple promesse d'embauche. La plupart des candidats au passage - qui proviennent désormais majoritairement du Mezzogiorno - seront régularisés a posteriori au centre de criblage de l'Office national de l'immigration (ONI) de Montmélian (Savoie), où viennent les recruter en camion les responsables des entreprises forestières de la région ${ }^{16}$. G. R., de Serra San Bruno (Calabre), témoigne de sa stratégie migratoire et professionnelle: "Je suis venu en France pour la première fois en 1955, avec un contrat de travail de saisonnier. J'ai d'abord été recruté comme câbliste dans les Alpes-Maritimes. Après, j'ai trouvé un patron dans le Var, à côté de Toulon. Un soir, au mois de novembre, on rencontre un type qui cherchait une équipe de câblistes. Moi, je voulais rester en France et avoir un contrat de travail à l'année. Je lui ai donné le nom de quatre ou cinq collègues qui venaient du même coin que moi. Alors on est allé passer la visite médicale obligatoire à l'ONI pour avoir le contrat. Les deux premières années où j'ai travaillé vers Nice, j’ai passé la visite au centre de Milan. Puis, quand jétais à Toulon, je l'ai Entre 1945 et 1950, ce sont des milliers de Transalpins qui franchissent illégalement la frontière alpine, avec ou sans le concours de passeurs, pour rejoindre l'Hexagone, munis parfois d'une simple promesse d'embauche. passée à Marseille. Alors, nous avons installé nos câbles dans les gorges du Verdon en 1957. On a ensuite coupé du pin un peu partout, jusqu'au bord de la plage à Cassis (Bouches-du-Rhône). Mais, là-bas, ceétait pénible, l'été, il faisait très chaud. On dormait dans des vieilles cabanes de chasseurs pleines de souris et on buvait de l'eau de citerne, c'était dégoûtant. Un de mes collègues qui, une année auparavant, avait travaillé dans le Vercors, a trouvé un patron à Autrans (Isère). On y est arrivé le 20 avril 1961. On a d'abord travaillé quelques mois entre hommes puis, comme je voulais rester en France, on a fait venir les femmes. Ensuite, j'ai travaillé pour la scierie Eymard qui m’a fait installer des câbles. J'ai travaillé comme ça pendant vingt-cinq ans un peu partout dans le Vercors. Sur le chantier, on était treize. Y a une équipe qui coupait tout le temps, après, nous on lançait les bois au pic et une autre équipe montait le téléphérique. On logeait 
dans des cabanes de l'ONF, des maisons forestières. L'hiver, je travaillais à la scierie à Villard-de-Lans. Ma femme faisait des ménages. On a aussi travaillé dans la Drôme pour les Papeteries de France. Je me suis arrêté en 1985, parce que j’ai eu un accident et que je me suis blessé au genou."

\section{Réseaux transnationaux de recrutement et dégradation des conditions de travail}

L'hégémonie des Italiens cesse dans le bûcheronnage au cours des années 1960. Nombre de ceux qui se sont installés en France montent alors leur propre affaire dans l'exploitation, le transport ou la transformation de la matière ligneuse. Cela montre à la fois leur spécialisation dans ce domaine d'activité et une ascension sociale rapide. À cette époque se diffusent tronçonneuses puis engins de débardage motorisés qui permettent aux responsables des coupes de solliciter une main-d'œuvre moins spécialisée, susceptible d'ap-

Les migrants seront

désormais employés

temporairement aux travaux d'abattage et de façonnage,

en particulier de petit bois

pour les papetiers et

les fabricants de panneaux

à particules

bûcherons français sont italiens, 7 \% espagnols, $3 \%$ portugais, yougoslaves et polonais. En 1970, les sources officielles indiquent désormais $35 \%$ de Portugais, 30\% d'Espagnols et, enfin, 35 \% d'Italiens, de Yougoslaves et de Nord-Africains ${ }^{17}$. À cette époque, on observe également l'introduction de bûcherons turcs dans les Alpes, les Pyrénées et surtout dans le Massif central ${ }^{18}$. La loi de 1974 sur l'immigration marque toutefois un arrêt brutal du recrutement des permanents en forêt. Les migrants seront désormais employés temporairement aux travaux d'abattage et de façonnage, en particulier de petit bois pour les papetiers et les fabricants de panneaux à particules. Ces entreprises recrutent des bûcherons salariés étrangers en contrat à durée déterminée via leurs sociétés d'approvisionnement. En 1984, dans l'Hexagone, l'exploitation forestière employait encore des effectifs importants d'étrangers, dont environ 6000 pour les seuls travaux de bûcheronnage, ce à quoi il conviendrait d'associer les activités connexes (sylviculture, débardage, scierie), soit encore $30 \%$ de l'effectif total des bûcherons.

De nos jours, à peine $4 \%$ des propriétaires forestiers sylviculteurs gèrent et exploitent leurs forêts à l'aide de leurs propres salariés. La grande majorité des travaux en forêt sont donc réalisés par des entreprises extérieures au territoire. Ce fut notamment le cas après les tempêtes de décembre 1999 qui, par leur violence exceptionnelle, nécessitèrent la mise en place de vastes chantiers, pour lesquels on a sollicité des étrangers non communautaires. Avec l'élargissement de l'Union européenne, l'emploi des étrangers en forêt prend de nouvelles formes liées à l'augmentation de la concurrence internationale et à la libéralisation des marchés. Dans les chantiers à grande échelle, sur fond de vieillissement (usure physique précoce) de la population ouvrière, on a fréquemment recours à de jeunes travailleurs immigrés particulièrement productifs, soumis à un "système d'autodiscipline collective ${ }^{19 "}$. 
Bûcherons calabrais, massif de la Chartreuse, $X X I^{e}$ siècle.

(c) Emmanuel Breteau

Dans l'équipe, il règne, en effet, une émulation de tous les instants, fondée sur la solidarité entre compatriotes et qui tire la productivité vers le haut. Le travail en exploitation forestière demeure une activité difficile à appréhender compte tenu de la dispersion des chantiers, mais aussi du fait d'une culture du secret très présente chez les protagonistes d'une activité exercée parfois aux limites de la légalité. L'inspection du travail constate, en effet, de plus en plus d'irrégularités dans le recrutement de la main-d'œuvre étrangère employée pour un travail harassant et dangereux, exercé six jours sur sept. Il peut s'agir de travail "dissimulé en forêt" ou d'absence de déclaration d'activité auprès des organismes sociaux et fiscaux. Les autorités découvrent régulièrement de nouvelles filières qui profitent de travailleurs étrangers, parfois à l'insu des exploitants forestiers : "Le 23 mai 2007, nous sommes informés par les services de I'ITEPSA de l'Isère (inspection du travail agricole), du contrôle de six bûcherons slovaques, salariés d'une entreprise slovaque. Ceux-ci semblent mis à la disposition des entreprises utilisatrices par une société implantée dans le département de Côte-d'Or." Des opérations de contrôle organisées par la Mutualité sociale agricole (MSA) et l'inspection du travail dans différents départements révèlent, en outre, des manquements à

la sécurité : défaut de "Les Turcs sont arrivés balisage des zones de aux Contamines (Chamonix) découpe, ouvriers sans en baskets, sans protection, tenue de protection, embauchésaunoirpar absence d'un person- un marchand de bois qui nel formé aux premiers a son recruteur. Ce dernier secours, ce qui contri- était venu les chercher bue à la multiplication sur Grenoble." des accidents. Ce dont témoigne un bûcheron de Haute-Savoie: "Les Turcs sont arrivés aux Contamines (Chamonix) en baskets, sans protection, embauchés au noir par un marchand de bois qui a son recruteur. Ce dernier était venu les chercher sur Grenoble. Ils habitaient dans une caravane [...]. Le plus vieux s'est fait écraser par un arbre pendant qu'il écorçait. Ils n'ont pas voulu le descendre en hélico, parce qu’il aurait fallu le déclarer ${ }^{20}$."

\section{Le bûcheron étranger dans la fiction : figure de la déviance ou nouveau prolétaire?}

L'isolement du bûcheron dans la sylve et son ardeur au travail ont favorisé de longue date la construction de stéréotypes véhiculés par le cinéma, la littérature ou les fêtes rurales, comme les concours de bûcherons. Nourri d'une certaine vision romantique exaltant la liberté, la force et l'adresse du "nomade des forêts", le cinéaste britannique Tony 
Richardson explore dans Mademoiselle (1966) le mal de vivre des sédentaires dans une bourgade de la Corrèze, que rejoignent au printemps des saisonniers italiens. "Un jour ici, le lendemain ailleurs", soupire envieux, le brigadier de gendarmerie. Leur présence semble se traduire par des actes déraisonnables (inondation d'une ferme, incendies pyromanes). Le coupable désigné à la vindicte populaire est un de ces bûcherons étrangers qui suscitent désir chez les femmes et haine chez les hommes. À travers la mise à mort de ce Malgré les progrès de bouc émissaire, Richardson la mécanisation, la récolte du bois dépend encore, particulièrement en montagne, de la maind'œuvre d'exploitation forestière. Il n'est donc pas rare d'apercevoir à l'automne des équipes de bûcherons d'Europe de l'Est. dénonce la xénophobie d'une certaine France rurale.

D'autres auteurs dotés d'une conscience sociale aiguë s'intéressent à l'expérience migratoire et aux conditions de travail en forêt. Dans un célèbre roman noir, Le Petit Bleu de la côte ouest (1967), Jean-Patrick Manchette narre les mésaventures de Gerfaut - antihéros traqué par des tueurs impitoyables - qui échoue, au terme d'une cavalcade dans les Alpes, près d'un camp de bûcherons portugais qui lui offrent l'hospitalité. Le sentiment de sécurité qu'il éprouve à leur contact témoigne bien de l'invisibilité du chantier. Sérieusement documenté, Manchette dénonce les conditions de vie de ce moderne prolétariat des forêts : "Ils campaient sous une grande bâche montée sur des pieux. Ils avaient des couvertures dégueulasses et des matelas de rameaux et de feuilles. Ils disposaient de pain rassis, d'un peu de vin d'Algérie, de fromage, de mauvais café, de plusieurs grands sacs de légumes secs et de trois revues pleines d'illustrations photographiques obscènes. Ils étaient équipés de haches, de scies et de deux tronçonneuses Homelite. Ils séjournaient illégalement en France, n'avaient aucune sorte de Sécurité sociale et touchaient un peu plus de la moitié du Smic pour un travail de soixante à soixante-dix heures par semaine."

\section{Conclusion}

Depuis le XIX siècle, les grands chantiers forestiers des Alpes et du Jura sont majoritairement exploités par des spécialistes étrangers. Si le charbonnier a bien disparu au début des années 1970, le bûcheron, entrepreneur ou salarié, demeure une figure incontournable du monde rural contemporain ${ }^{21}$. Malgré les progrès de la mécanisation, la récolte du bois dépend encore, particulièrement en montagne, de la main-d'œuvre d'exploitation forestière. Il n'est donc pas rare d'apercevoir à l'automne des équipes de bûcherons d'Europe de l'Est. Comme les Italiens naguère, ces bûcheronscâblistes - recrutés et dirigés par un contremaître installé en France - sont à la tâche de l'aube au couchant et vivent dans des habitats précaires à proximité du chantier.

Aux côtés du fromager et du berger, le "cueilleur d'arbres" incarne le pittoresque montagnard. Gardons-nous cependant d'esthétiser cette profession difficile nécessitant la connaissance du milieu végétal et la maîtrise d'une technique dont les migrants saisonniers furent et sont encore détenteurs. 